directly to detectors. Some demultiplexing is acceptable in less demanding applications where many similar items of equipment are controlled, e.g. power supplies. Here, a field bus controller drives a multidrop cable connecting up to 30 equipment control assemblies (ECA's) implemented using the MIL-STD-1553-B standard field bus. The ECA's, located close to the subsystems they control, consist of G64 or VMEbus crates with microprocessors.

\section{Concluding Remarks}

Standardization of bus systems has allowed investments in design, material, manpower and technical know-how to be capitalized upon. Meanwhile, the diversity of applications, users' experience and contributions to international standards bodies have enhanced and optimized technical specifications.

One consequence is a change-over from the use of buses for simple input/output functions to the complete integration of all the operations required for data acquisition and process control. However, comparisons between bus systems used for controlling large accelerators suggest that the basic requirements have not changed drastically in 20 years: distributed communication, local processing power, remote control and surveillance, and local interaction with graphics remain essential.

The revolution is that these functions are now implemented more quickly, economically and flexibly, and with greater pre-processing of data. The integration of complete functions in a single chip (i.e. network protocols, processors, memories, digital signal processors, peripheral controllers, intelligent sensors, etc.) and the standardization of hardware for bus systems means that more computer power and processing functions can now be packed into a single multiprocessor VMEbus crate than in a complete 1970's style front-end process control assembly requiring several racks of equipment.

Further improvements in software, standardization of software tools, languages, real-time kernels, communication packages, graphics packages, etc. are essential to improve the efficiency of process control. For instance, we dream of software modules plugging into a standard software system (the "software bus") much like a VMEbus module plugs into a standard VMEbus. Moreover, we are already not far from a UNIX real-time software kernel capable of dynamic load-sharing among several identical VMEbus processors in a crate and remotely communicating with user-friendly workstations for the operators.

\section{REFERENCES}

[1] EUR 11079: Guide to Internationally Recognized Hardware Interfaces (CEC, Brussels) 1988.

[2] Altaber J. et al., Proc.1983 Particle Accelerators Conference, IEEE Trans. NS-30 (1983).

\title{
CIM For Particle Factories
}

Large experimental facilities may seem unusual to the average person, but they are comparable to industrial machines suggesting an industrial approach for integrating computers. A major installation such as an accelerator complex or "particle factory" is mostly made up of well-defined industrial components so its operation largely leans on functional blocks similar to those found in industry.

But this is not the generally accepted view. The problems of pioneering in statistics-based research topics such as particle physics lead engineers and physicists to work at the limits of what is possible in several fields (e.g. electronics, mechanics, computing, materials, etc.). They are forced to permanently explore novel ideas and to evaluate and apply promising new technologies as soon as they emerge from laboratories. This feature also pushes them into initiating new developments until they begin to feel they have to step beyond in dustry. One finally becomes accustomed to developing everything that is needed be cause one generally achieves better results than with what is easily available.

This has been the case on introducing computers into experimental facilities. There has been an anarchic development of tools for engineering, production, control, planning and administrative support activities. Isolation from developments elsewhere and the lack of defined goals rapidly became the main problems. The software industry was in the meantime developing products for a much larger community.

\section{Exploiting CIM}

The spectacular progress of informatics has generated considerable interest in attempts to model a commercial enterprise. Views of an experimental facility, notably a particle factory, that are developed using some of the latest descriptions [1, 2] emphasize two unique features:

- the facility does not sell, strictly speaking, the products it manufactures;

- products such as high energy particles cannot be stored or transported in a conventional way as these operations need complex machines. The products are instead manufactured for local use by physicists.

Considering an experimental facility as a manufacturing site nonetheless provides a working framework for achieving greater efficiency. A computer-integrated manufacturing (CIM) system should thus be considered for an experimental facility as reports show that its implementation produces encouraging results $[3,4]$ by generating major improvements in productivity, cost savings, throughput and flow control.

Although CIM is not yet clearly defined [3] it aims to integrate computers and adequate software tools, which may not necessarily exist as yet, into all aspects, including management, of a manufacturing cycle (planning, design, etc.). A top-down approach to implementing CIM leans on two fundamental pillars: communication and data exchange and management. Success requires:

- A coherent and powerful communication network based on international standards that links together all laboratories, offices, workshops, accelerators and experiments. Organized into interconnected islands of local area networks (LAN's), the overall network allows for an independent and flexible selection of computer-based products aimed at helping workers.

- Computer-based software tools that comply fully with international data exchange standards.

- A single, widely accessible database management system (DBMS) for the collection and retrieval of data originating from different sources (administrative, technical, etc.) or having different natures (text, picture, sound, etc.).

Weaknesses in any of these areas result in isolated islands of different computerbased tools to aid the different groups (systems designers, operators, etc.) and a large reduction in the overall benefits. A correctly configured CIM system can help by acting as the glue between individual tools used for different functions in a company [5].

CERN is an example of one research organization that is currently working towards integration. A powerful communication network has been implemented, with Ethernet and token rings as wide area networks and with Ethernet and communication systems based on the MIL-1553-B standard for LAN's. A DBMS has been approved for both process control and administrative activities. Software tools are starting to be connected and although links are missing (mainly in administrative and support areas), an ambitious project to integrate all administrative tasks is underway.

\section{Conclusions}

Large experimental facilities in physics have enjoyed the advantage of an intimate contact with computers. But the complex, slightly anarchic situation which grew up should not divert attention from the coherence that is now emerging elsewhere. It is essential that managers of these centres should now seek this coherence when approving computer-based software products. The application of full integration based on the CIM top-down approach applied to an experimental facility could then serve as a model for European industry.

\section{REFERENCES}

[1] Kosankc K. and Vlietstra J., Proc. 6th Ann. ESPRIT Conf., Brussels (1989).

[2] Playoust P., Tech. de I'Ingénieur, H6050. [3] Foltz M.T. and Tanik N.M., Int. J. Computer Applns. in Technology 3 (1990) 26.

[4] Costea I., Information Strategy, Summer issue (1990).

[5] Leonard R., Int. J. Computer Integrated Manufacturing I (1990) 13.

M. Rabany CERN, Geneva 